PROCEEDINGS OF THE

AMERICAN MATHEMATICAL SOCIETY

Volume 132, Number 5, Pages 1251-1255

S 0002-9939(03)07198-3

Article electronically published on October 9, 2003

\title{
CONTRACTIBLE FRÉCHET ALGEBRAS
}

\author{
RACHID EL HARTI
}

(Communicated by Joseph A. Ball)

\begin{abstract}
A unital Fréchet algebra $A$ is called contractible if there exists an element $d \in A \hat{\otimes} A$ such that $\pi_{A}(d)=1$ and $a d=d a$ for all $a \in A$ where $\pi_{A}: A \hat{\otimes} A \rightarrow A$ is the canonical Fréchet $A$-bimodule morphism. We give a sufficient condition for an infinite-dimensional contractible Fréchet algebra $A$ to be a direct sum of a finite-dimensional semisimple algebra $M$ and a contractible Fréchet algebra $N$ without any nonzero finite-dimensional two-sided ideal (see Theorem 1). As a consequence, a commutative lmc Fréchet $Q$-algebra is contractible if, and only if, it is algebraically and topologically isomorphic to $\mathbb{C}^{n}$ for some $n \in \mathbb{N}$. On the other hand, we show that a Fréchet algebra, that is, a locally $C^{*}$-algebra, is contractible if, and only if, it is topologically isomorphic to the topological Cartesian product of a certain countable family of full matrix algebras.
\end{abstract}

It is well known that in the finite-dimensional case, a complex algebra is contractible (separable) if, and only if, it is a semisimple algebra [7. An infinitedimensional contractible Banach algebra has yet to be found. In the Fréchet algebra case, there exist some infinite-dimensional contractible algebras. Hence, it is very important to study contractible Fréchet algebras, which are useful as a class of topological algebras. We begin by introducing the concept of Fréchet modules, and we recall some results on projective Fréchet modules. Later, we prove some results concerning a class of contractible Fréchet algebras.

A Fréchet space is a complete metrizable locally convex complex space. An algebra $A$ will be called a Fréchet algebra if $A$ is a Fréchet space with jointly continuous multiplication. For any two spaces $X$ and $Y$, we write $X \hat{\otimes} Y$ for the completed projective tensorial product [3]. Let $A$ be a unital Fréchet algebra. Following ([4], [5]), we define a Fréchet left $A$-module $X$ to be a Fréchet space that is also a unital left $A$-module such that the linear map $A \hat{\otimes} X \rightarrow X, a \otimes x \rightarrow a x$, is continuous. Right modules are defined analogously. A Fréchet $A$-bimodule is a Fréchet space with structural $A$-bimodule such that the linear map $A \hat{\otimes} X \hat{\otimes} A \rightarrow X$, $a \otimes x \otimes b \rightarrow a x b$ is continuous. A Fréchet $A$-bimodule $\mathrm{X}$ is said to be projective if the canonical morphism $\pi_{X}: A \hat{\otimes} X \hat{\otimes} A \rightarrow X$ has a right inverse Fréchet $A$-bimodule morphism. A morphism $\tau$ in the category of Fréchet modules is called $\mathbb{C}$-split if its kernel and its image both have a direct complement as Fréchet subspaces.

Received by the editors March 14, 2002 and, in revised form, January 8, 2003.

2000 Mathematics Subject Classification. Primary 13E40, 46H05, 46J05, 46 K05. 
Proposition 1 (see [4). Let $A$ be a unital Fréchet algebra. Then a Fréchet $A$ bimodule $X$ is projective if, and only if, for any Fréchet $A$-bimodule $Z$, every $\mathbb{C}$ split A-bimodule epimorphism $\tau: Z \rightarrow X$ has a right inverse Fréchet A-bimodule morphism.

For a unital Fréchet algebra $A$, a diagonal of $A$ is an element $d \in A \hat{\otimes} A$ such that $\pi_{A}(d)=1$ and $a d=d a \quad \forall a \in A$.

Definition. A unital Fréchet algebra is said to be contractible if it has a diagonal element.

The algebra $\mathbb{M}_{n}(\mathbb{C})$ of all $n \times n$ complex matrices has a diagonal and thus is contractible. Its diagonal is the element $\frac{1}{n} \sum e_{i j} \otimes e_{j i}$ where $\left(e_{i j}\right)_{1 \leq i, j \leq n}$ is the canonical base. A finite-dimensional and semisimple algebra is contractible, and it is isomorphic to the direct sum of a certain finite family of full matrix algebras. It was frequently asked in [4] if every contractible Banach algebra is a finite direct sum of full matrix algebras. Now consider the algebra $\mathbb{C}^{\mathbb{N}}$ of all infinite sequences of complex numbers under coordinate-wise multiplication with the canonical base $\left(e_{i}\right)_{i \geq 0}$. It is an infinite-dimensional, semisimple and contractible Fréchet algebra. The element $d=\sum_{i, j=1}^{\infty} \delta_{i j} e_{i} \otimes e_{j}$ is its diagonal, where $\delta_{i j}$ is the Kronecker delta. Moreover, a Cartesian product of any family of full matrix algebras is contractible. Notice that all examples here are semisimple.

Question 1. Is every contractible Fréchet algebra semisimple?

In fact, the converse is false: The algebra $C^{\infty}[0,1]$ is a unital semisimple Fréchet algebra but it is not contractible (see Theorem 2). In the terminology of homological algebras, a contractible (separable or finite-dimensional semisimple algebra) algebra $A$ means that $A$ is a projective $A$-bimodule. The following result is an adaptation of this.

Proposition 2 (44). For a unital Fréchet algebra A, the following statements are equivalent:

1. A is contractible.

2. $A$ is a projective Fréchet $A$-bimodule.

3. $A \hat{\otimes} A \rightarrow A$ has a right inverse Fréchet A-bimodule morphism.

4. For each Fréchet $A$-bimodule $X$, every derivation $D: A \rightarrow X$ from $A$ to $X$ is inner.

Proposition 3. Let $\theta: A \rightarrow B$ be a unital algebra morphism with $\theta(A)$ dense in $B$. If $A$ is contractible, then $B$ is also.

Proof. Applying Proposition 1 and Proposition 2, this result follows directly from the fact that every Fréchet $B$-bimodule is a Fréchet $A$-bimodule and every Fréchet $A$-bimodule morphism is a Fréchet $B$-bimodule morphism.

Proposition 4 (4]). Let I be a closed two-sided (or left) ideal in the contractible Fréchet algebra. If I is complemented in $A$ as a Fréchet subspace of $A$, then there exists in A another closed two-sided (or left) ideal $J$ such that $A=I \oplus J$.

Remarks. Let $A$ be a contractible Fréchet algebra. Then:

1. Obviously, for every finite-dimensional two-sided ideal (or left) $I$ of $A$, there exists a closed two-sided (or left) ideal $J$ such that $A=I \oplus J$. 
2. It is clear that every finite-dimensional minimal two-sided ideal (respectively left ideal) is isomorphic to a simple Fréchet $A$-bimodule (respectively left module).

3. Finally, every finite-dimensional two-sided ideal (respectively left ideal) is a direct sum of minimal two-sided ideals (respectively left ideals).

Now let $N=\{a \in A: a I=0$, for all finite-dimensional left ideals $I\}$. Then $N$ is a closed two-sided ideal.

Theorem 1. Let $A$ be a contractible Fréchet algebra. Then we have:

(1) $\overline{\operatorname{Rad}(A)} \subseteq N$ where $\operatorname{Rad}(A)$ is the Jacobson radical of $A$.

(2) If $A / N$ is a finite-dimensional algebra, then $N$ is contractible with no finitedimensional two-sided ideal and there are $n_{1}, \ldots, n_{k}$ in $\mathbb{N}$ such that

$$
A=N \oplus \mathbb{M}_{n_{1}}(\mathbb{C}) \oplus \mathbb{M}_{n_{2}}(\mathbb{C}) \oplus \ldots \oplus \mathbb{M}_{n_{k}}(\mathbb{C}) .
$$

Proof. 1. Because $N$ is closed, we have to show only that $\operatorname{Rad}(A) \subseteq N$. Let $a \in \operatorname{Rad}(A)$, and let $I$ be a finite-dimensional left ideal. Using the last remark, $A=I \oplus J$ for some left ideal. If $I$ is minimal, then $a I=0$, and so $a \in N$. If not, by the remark, $I$ is a direct sum of minimal left ideals and also $a I=0$.

2. In this case, there is a finite-dimensional two-sided ideal $M$ such that $A=$ $N \oplus M$. By Proposition $3, N$ is contractible. On the other hand, notice that a Fréchet space $X$ is a finite-dimensional $N$-bimodule if, and only if, it is a finitedimensional $A$-bimodule. Moreover, every finite-dimensional two-sided ideal of $N$ is isomorphic to a finite-dimenional $N$-bimodule. It follows that $N$ is contractible with no finite-dimensional two-sided ideal. It remains to show that $M$ is semisimple. Let $b \in \operatorname{Rad}(M)$. Then $a b$ is quasi-invertible for all $a \in M$ if, and only if, $a b$ is quasi-invertible for all $a \in A$. Therefore, $b \in \operatorname{Rad}(A)$ and so, $\operatorname{Rad}(M) \subseteq \operatorname{Rad}(A)$. Using 1, we have $\operatorname{Rad}(M)=0$.

Corollary. Let $A$ be a contractible Fréchet algebra such that every maximal left ideal is finite codimensional. Then $N=\operatorname{Rad}(A)$. Moreover, if $A / N$ is a finitedimensional algebra, then $A$ is a finite-dimensional semisimple algebra.

Proof. Let $a \in N$, and let $R$ be a maximal left ideal. Since $R$ is a finite-codimensional left ideal, there exists a finite-dimensional left ideal $J$ such that $A=R \oplus J$. Write $a=a e_{R}+a e_{J}$ where $e_{R}$ and $e_{J}$ are the components of the identity. Since $a e_{J}=0$, $a \in R$. Therefore, $a \in \operatorname{Rad}(A)$ and so, $N \subseteq \operatorname{Rad}(A)$. By the first statement of Theorem 1, we have $N=\operatorname{Rad}(A)$. If $A / N$ is finite-dimensional, then using statement (2) of Theorem 1, we have $A=\operatorname{Rad}(A) \oplus M$ with $M$ as above. This is possible only if $\operatorname{Rad}(A)=0$. This completes the proof.

A Fréchet algebra $A$ is called an lmc Fréchet algebra if its topology is induced by a separating directed set of algebra seminorms $\left(\|\|_{i}, i \in I\right.$ ) (see [8], [9]). It is called a Fréchet $Q$-algebra when its group of invertible elements is an open set.

Proposition 5. If $A$ is a contractible commutative lmc Fréchet Q-algebra, then $A / N$ is a finite-dimensional algebra.

Proof. In this situation (see [11]), every character (or complex homomorphism) $\chi: A \rightarrow \mathbb{C}$ is continuous and its kernel is a (closed) finite-codimensional maximal ideal. Moreover, every maximal ideal is the kernel of a character. We denote by $X_{A}$ the set of maximal ideals in $A$ with Gelfand topology (if $x \in A$ and $M=\operatorname{ker} \chi \in X_{A}$ where $\chi$ is a character, set $\hat{x}(\chi)=\chi(x)$. The Gelfand topology is the weakest for which $\hat{x}$ is continuous on $\left.X_{A}\right)$. Then $X_{A}$ is a compact set. Notice that $A / N$ is also 
a contractible lmc Fréchet $Q$-algebra (see Proposition 3 and [11]). For each $J$ in $X_{A / N}$, there is a finite-dimensional ideal $I$ in $A / N$ such that $A / N=J \oplus I$. Then $\operatorname{hull}(I)^{c}=X_{A / N} \backslash \operatorname{hull}(I)=\{J\}$ where $\operatorname{hull}(I)=\left\{M \in X_{A / N}: I \subseteq M\right\}$. Indeed, let $R \in \operatorname{hull}(I)^{c}$. Then $A=I+R$. Put $e=i+r$ with $i \in I$ and $r \in R$. Note that $e=e_{I}+e_{J}$ with $e_{I} \in I$ and $e_{J} \in J$. Then $e_{J}=i e_{J}+r e_{J}$. Since $i e_{J}=0$, we see that $e_{J} \in R$. Therefore, $J \subseteq R$, and so $J=R$. It is easy to see that $J \in h u l l(I)^{c}$. Then for each $J$ in $X_{A / N},\{J\}$ is an open set. Since $X_{A / N}$ is compact, it is a finite set. On the other hand, we can see, as in the proof of the corollary, that $N=\operatorname{Rad}(A)$. Therefore, $A / N$ is a semisimple commutative lmc Fréchet $Q$-algebra with finite Gelfand spectrum. It is necessarily a finite-dimensional algebra.

Theorem 2. A commutative lmc Fréchet Q-algebra is contractible if, and only if, it is isomorphic to $a \mathbb{C}^{n}$.

Proof. By Proposition 5 and the corollary, we deduce this result.

Question 2. Does there exist an infinite-dimensional contractible Fréchet $Q$ algebra?

Proposition 6. Let $A$ be a contractible Fréchet $Q$-algebra. If $A$ is not semisimple, then the Jacobson radical Rad $(A)$ does not have a complementary space in $A$.

Proof. It is well known that the Jacobson radical $\operatorname{Rad}(A)$ of a Fréchet $Q$-algebra $A$ is closed. Assume that $\operatorname{Rad}(A)$ has a Fréchet space complement in $A$. Because $A$ is contractible, there exists a closed ideal $J$ of $A$ such that $A=\operatorname{Rad}(A) \oplus J$. This is possible only if $\operatorname{Rad}(A)=\{0\}$.

A Fréchet locally $C^{*}$-algebra is an lmc Fréchet algebra with its topology induced by a countable separating directed set of algebra $C^{*}$-seminorms $\left(\|\|_{n}, n \in \mathbb{N}\right)$.

Theorem 3 ([2]). A Fréchet locally $C^{*}$-algebra $A$ is contractible if, and only if, it is topologically isomorphic to a topological product of full matrix algebras.

Proof. It is clear that a locally $C^{*}$-algebra $A$ isomorphic to a product of a family of full matrix algebras, has a diagonal, and so, it is contractible.

Conversely, let $A$ be a contractible locally $C^{*}$-algebra. Note that $A$ is the inverse limit of a countable system of $C^{*}$-algebras $\left(A_{n}, n \in \mathbb{N}\right.$ ) (see [6] and [9]). By Proposition 3 , each $A_{n}$ is a contractible $C^{*}$-algebra. Using [10, $A_{n}$ is finitedimensional and so, it is isomorphic to a finite direct sum of certain full matrix algebras $\bigoplus_{k_{n} \in F_{n}} \mathbb{M}_{k_{n}}(\mathbb{C})$. Put $\sigma=\bigcup F_{n}$. Using [1, page 80, Proposition 3] and [8, Lemma XII.2.1], we have

$$
\lim _{\leftarrow}\left(\bigoplus_{k_{n} \in F_{n}} \mathbb{M}_{k_{n}}(\mathbb{C})\right)=\Pi_{k \in \sigma} \mathbb{M}_{k}(\mathbb{C}) .
$$

This completes the proof.

\section{ACKNowledgement}

I wish to express my sincere thanks to Professor A. Ya. Helemskii for his help and the various instructive and penetrating discussions. 


\section{REFERENCES}

[1] N. Bourbaki, Théorie des ensembles, Chapitre 3, Hermann, Paris, 1963. MR 27:4758

[2] Maria Fragoulopoulou, Structure of contractible locally $C^{*}$-algebras, Proc. Amer. Math. Soc. 129 (2001), 2889-2896. MR 2002c:46106

[3] A. Grothendieck, Produits tensoriels topologiques et espaces nucléaires, Mem. Amer. Math. Soc. 16 (1955), 140 pp. MR 17:763c

[4] A. Ya. Helemskii, The Homology of Banach and Topological Algebras. Kluwer Academic Press, Dordrecht, 1989. MR 92d:46178

[5] A. Ya. Helemskii, Banach and locally convex algebras, The Clarendon Press, Oxford University Press, New York, 1993. MR 94f:46001

[6] A. Inoue, Locally $C^{*}$-algebra, Mem. Fac. Sci. Kyushu Univ. Ser. A 25 (1971), 197-235. MR 46:4219

[7] M. J. Liddell, Separable topological algebras I, Trans. Amer. Math. Soc. 195 (1974), 31-59. MR 50:5471

[8] A. Mallios, Topological Algebras. Selected Topics, North-Holland, Amsterdam, 1986. MR 87m:46099

[9] E. A. Michael, Locally multiplicatively-convex topological algebras, Mem. Amer. Math. Soc. No. 11 (1952), 82 pp. MR 14:482a

[10] Ya V. Selivanov, Some questions on the homological classification of Banach algebras, Izdat. Moskov. Univ., Moscow (1978).

[11] W. Zelazko, On maximal ideals in commutative $m$-convex algebras, Studia Math. 58 (1976), 291-298. MR 55:8803

University Hassan I, Department of Mathematics, FSt of Settat, BP 577, Settat, MOROCCO

E-mail address: elharti@uh1.ac.ma 